\title{
Endovascular Management of Massive Hematemesis due to Aorto-Esophageal Fistula
}

\author{
Yugandhar Samireddypalle ${ }^{1}$ Amitha Vikrama K. S. \\ ${ }^{1}$ Department of Interventional Radiology, Sakra World Hospital, \\ Bangalore, Karnataka, India
}

J Clin Interv Radiol ISVIR 2019;3:67-68

A 75-year-old man presented with weight loss, left hip pain, and dysphagia of 1-month duration. He has prior history of well-controlled diabetes and hypertension. He was clinically stable at the time of presentation with blood pressure (BP) of $160 / 100 \mathrm{~mm} \mathrm{Hg}$ and heart rate (HR) of 82 beats/ min. A radiograph of the pelvis demonstrated pathologic fracture of the left iliac bone. Contrast-enhanced computed tomography (CT) of the abdomen revealed multiple necrotic lower mediastinal nodes. During the hospital stay, there was a sudden episode of hypotension (BP of $80 / 60 \mathrm{~mm} \mathrm{Hg}$ ), tachycardia, tachypnea, and desaturation on room air. The patient was moved to the intensive care unit (ICU) and resuscitated. Hemoglobin decreased from 12 to $7 \mathrm{~g} /$ $\mathrm{dL}$. While the patient was being moved to the CT scanner, he had a sudden episode of massive hematemesis. CT angiography of the chest demonstrated diffuse irregular circumferential mural thickening of the thoracic esophagus from D3 to D8 level with extravasation of the contrast material into the esophageal lumen (-Fig. 1A, B). A nipple was noted in the anterior wall of the thoracic aorta at the level of D5 vertebral body ( - Fig. 1C). Based on these findings, aortoesophageal fistula was diagnosed.

\begin{abstract}
Address for correspondence Yugandhar Samireddypalle, MBBS, DNB, PDCC, Department of Interventional Radiology, Sakra World Hospital, Bangalore 560103, Karnataka, India (e-mail: yugu.samireddypalle@gmail.com).
\end{abstract}

Endovascular management was planned for immediate treatment of the aortoesophageal fistula. Aortography from a left femoral arterial access did not reveal any obvious extravasation. Mild luminal narrowing was seen at the level of isthmus (arrows). A correlative perioperative endoscopy was performed in the angiography suite that confirmed presence of extensive intraluminal esophageal blood clots at D3 level. Right femoral arterial cutdown was performed. A 30- × 30- × 80-mm stent graft system (Ankura; Lifetech Scientific) was advanced into the arch of aorta beyond the left subclavian artery (LSCA) over a stiff guidewire positioned within the ascending aorta ( - Fig. 2A). The uncovered portion of the stent was deployed to extend beyond the LSCA ostium, and the covered portion of the stent extended across $70 \%$ of the ostium of the LSCA ( - Fig. 2B). The distal portion of the stent was deployed at the level of inferior margin of D6 vertebra. Poststent aortography showed good antegrade flow across the graft without any extravasation. There was mild delayed but persistent antegrade flow through

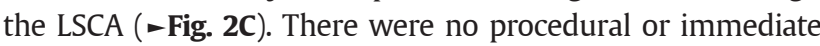
postprocedural complications.

Endoscopic evaluation the following day revealed ulceroproliferative tumor growth, and biopsy confirmed

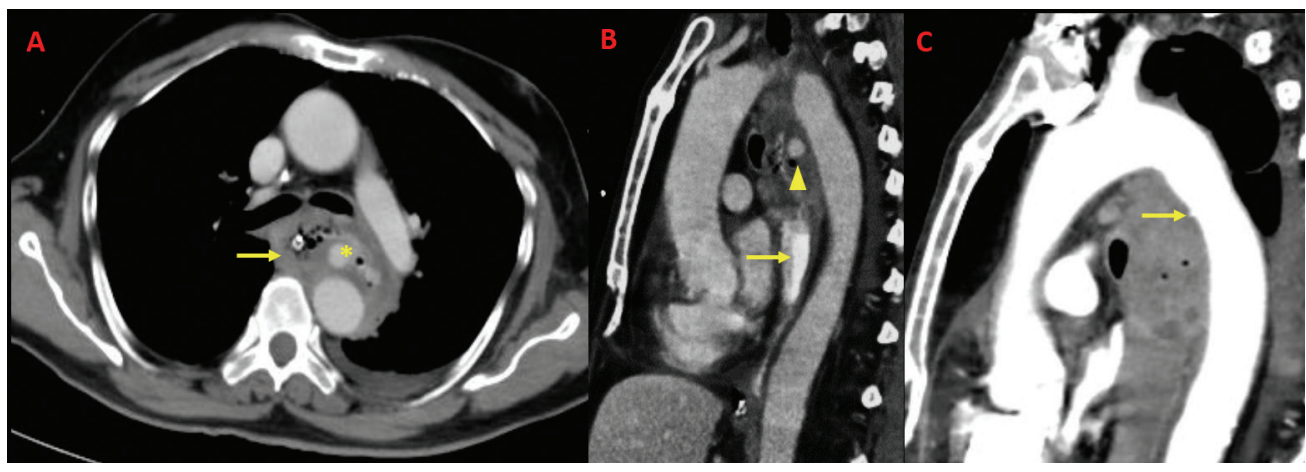

Fig. 1 (A) Axial contrast-enhanced CT at the level of carina demonstrating irregular circumferential mural thickening of the thoracic oesophagus (arrow) with obvious extravasation of contrast from the aorta (asterisk). (B) Sagittal reformatted CT image demonstrates the fistulous tract (arrow head) with opacification of the esophagus. The extravasated contrast is noted in the lower thoracic esophagus (arrow). (C) Sagittal CT aortogram image demonstrating a focal nipple of the anterior aortic wall at the level of D5 vertebra (arrow). There is also heterogeneous soft tissue mass in the esophagus with few air pockets within.

received

September 18, 2018 accepted after revision

November 13, 2018

published online

April 18, 2019
DOI https://doi.org/

$10.1055 / \mathrm{s}-0039-1681120$

ISSN 2457-0214.
(C2019 by Indian Society of Vascular and Interventional Radiology
License terms

() (1) $\Theta \circledast$ 


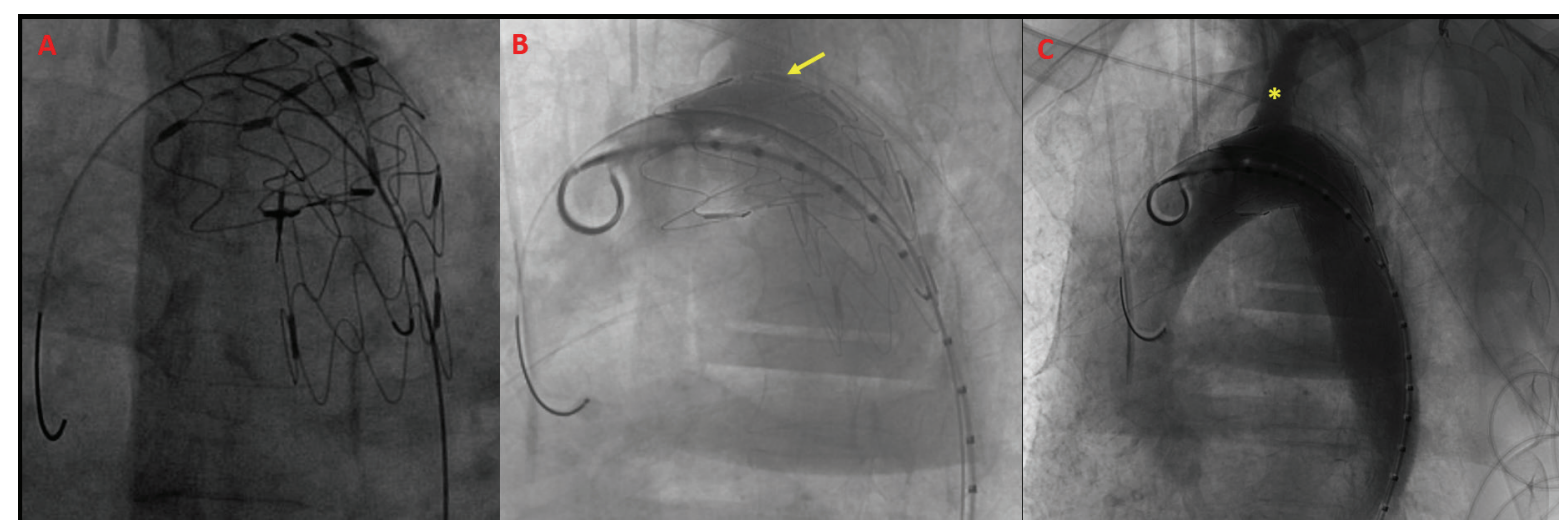

Fig. 2 (A) A 30- $\times$ 30- $\times$ 80-mm Ankura TAA stent graft system from Lifetech was placed across the proximal descending thoracic aorta, proximally from the LSCA ostium up to the level of inferior border of D6 vertebra. (B) The ostium of the LSCA was partially occluded by the covered portion of the stent graft (indicated by the arrow). (C) Postdeployment check digital subtraction aortogram showing normal filling of the aorta and left subclavian artery (asterisk) with no obvious contrast extravasation.

esophageal squamous cell carcinoma. The patient had a few episodes of melena for up to 48 hours. However, there was no change in hemoglobin. Esophageal stenting was deferred in view of reduced life expectancy due to advanced malignancy. A feeding gastrostomy tube was placed. The patient was hemodynamically stable. After 3 weeks, he developed aspiration pneumonia and eventually died due to uncontrolled septicemia.

\section{Discussion}

Aortoesophageal fistula is an uncommon cause of lifethreatening hematemesis. ${ }^{1}$ Thoracic aortic aneurysm is the most common cause of the fistula, ${ }^{1}$ followed by foreign-body ingestion and esophageal malignancy. ${ }^{1}$ Chiari described aortoesophageal syndrome, which comprises a sequence of symptoms such as chest pain followed by a asymptomatic "latent period," a prodromal hemorrhage, followed by fatal exsanguination. ${ }^{2}$

Conventional management includes thoracotomy and aortic repair surgery, which has high morbidity and mortality. Endovascular management of aortoesophageal fistula through thoracic endovascular aortic repair (TEVAR) has emerged as an effective alternative, especially as a bailout procedure. Some recommend it as a bridge procedure, followed by elective definitive aortic repair at a later date. ${ }^{3}$ TEVAR provides immediate control of life-threatening bleeding. However, the main postprocedural complication is mediastinitis due to ooze from the esophageal rent, which may progress to stent graft infection. TEVAR can be used as a definitive procedure, if infection can be controlled medically or with surgical debridement. ${ }^{4}$ Allen and Sebastian ${ }^{5}$ proposed that TEVAR should be done in conditions with low risk of infection such as infection with low virulent organisms, absence of gross purulence, and high operative risk. The role of esophageal stenting is unclear; however, there is definite potential of reducing the infection of the aortic stent graft by preventing esophageal leak-related mediastinitis.

\section{Informed Consent Statement}

Written informed consent was obtained from all patients for publication of this case report, including accompanying images.

\section{Conflict of Interest \\ None}

\section{References}

1 Flores J, Shiiya N, Kunihara T, Yoshimoto K, Yasuda K. Aortoesophageal fistula: alternatives of treatment case report and literature review. Ann Thorac Cardiovasc Surg 2004;10(4):241-246

2 Chiari HU. Fremdkorpeverletzung des oesophagus mit aortenperforation. Ber Klin Wochenschr 1914;51:7-9

3 Burks JA Jr, Faries PL, Gravereaux EC, Hollier LH, Marin ML. Endovascular repair of bleeding aortoenteric fistulas: a 5-year experience. J Vasc Surg 2001;34(6):1055-1059

4 Liang H, Chen C, Liu W, Yu F. Definitive treatment for aortoesophageal fistula by endovascular stent graft. Indian J Surg 2016;78(2):151-154

5 Allen RC, Sebastian MG. The role of endovascular techniques in aortoesophageal fistula repair. J Endovasc Ther 2001;8(6):602-603 